\section{Alcohol and death certification: a survey of current practice and attitudes}

Official estimates of all deaths from cirrhosis in England and Wales are lower than those in other countries with similar levels of alcohol consumption. ${ }^{1}$ In addition, officially recorded death rates from alcoholic cirrhosis appear lower than rates suggested by results of clinical surveys. ${ }^{2}$ These discrepancies are thought to be due largely to underrecording on death certificates, perhaps to avoid the statutory obligation (waived in July 1984) to report deaths related to alcohol to the coroner. ${ }^{3}$ We have examined the recording of alcohol abuse on death certificates and factors influencing such recording.

\section{Methods and results}

All 125 death certificates signed by the 43 preregistration housemen at Middlesex and University College Hospitals over a three month period were examined. Those with a diagnosis in which alcohol may have been aetiologically important $(n=40)$ were selected for review of the case notes (table). Thirty eight case notes were available and were reviewed $(a)$ to establish the accuracy of certification with special reference to alcohol and $(b)$ to assess the adequacy of the history of alcohol consumption recorded.

Diagnoses on death certificates selected for review of case notes

\begin{tabular}{lc}
\hline \multicolumn{1}{c}{ Diagnosis } & No of cases \\
\hline Cirrhosis of the liver & \\
(two patients certified as alcoholic) & 4 \\
Carcinoma of the tongue & 2 \\
Carcinoma of the oesophagus & 2 \\
Carcinoma of the stomach & 5 \\
Carcinoma of the pancreas & 5 \\
Cardiovascular diseases & 17 \\
Cerebrovascular diseases & 5 \\
\hline
\end{tabular}

The word alcoholic appeared on only two certificates; these were two of the four patients with a diagnosis of cirrhosis of the liver. All four, however, had established alcoholic liver disease. Two other patients, both with carcinoma of the tongue, had an alcohol intake of more than 100 units a week. Eight case notes were excluded from the study because patients had been too ill to give a history or a standardised history sheet had been used. Of the remaining 30 case notes, 17 $(57 \%)$ gave a quantitative assessment of alcohol consumption, seven gave qualitative comments, and six gave no history.

A self administered questionnaire covering $(a)$ possible reasons for not recording alcohol abuse on death certificates and whether such recording was considered important, $(b)$ knowledge of the current coroners' rules concerning alcohol related deaths, and $(c)$ the frequency of recording alcohol consumption was answered by 38 of the 43 housemen. The same questionnaire was also answered by 35 of 49 general practitioners in the Aylesbury Vale district of Buckinghamshire, who were selected as a comparison group.

Ninety per cent of both housemen and general practitioners (35 and 32, respectively) considered it important to record alcohol abuse on death certificates. Almost half the doctors in both groups admitted, however, that they would record it in only $50 \%$ of relevant cases. Among housemen this reluctance was related mainly to a lack of firm evidence of the aetiological importance of alcohol in individual cases, although nine $(31 \%)$ also believed that the stigma of alcoholism or the risk of distressing relatives might influence their recording. This number rose to $18(51 \%)$ among general practitioners $\left(\chi^{2}=4 \cdot 88, \mathrm{df}=1\right.$, p $<0.05$ ). Less than $20 \%$ in each group were aware of the current coroners' rules. Almost half the housemen were ignorant of such rules, whereas two thirds of general practitioners thought that the old rules still applied. All but one houseman claimed "almost always" to record details of alcohol consumption routinely, whereas only $21(60 \%)$ general practitioners thought they did so $\left(\chi^{2}=13 \cdot 38, \mathrm{df}=1, \mathrm{p}<0 \cdot 001\right)$.

\section{Comment}

These results confirm a reluctance to record alcohol abuse on death certificates, despite a general acknowledgment of the importance of doing so. Although housemen claimed "almost always" to record alcohol consumption routinely, almost half the case notes contained inadequate information. Nevertheless, this represents a $50 \%$ improvement compared with a similar study six years ago. ${ }^{4}$ Ignorance about current coroners' rules remains widespread and may influence recording by both general practitioners and housemen. Routine recording of alcohol consumption in case notes and of alcohol abuse on death certificates must be encouraged to enable accurate statistics relating to alcohol to be generated and used in health care planning.
We thank the house officers and the general practitioners who completed the questionnaire.

1 Taylor D. Alcohol: reducing the harm. London: Office of Health Economics, 1981.

2 Morgan MY. Alcohol as a cause of liver disease in the United Kingdom. In: Hall P, ed. Alcoholic liver disease: pathobiology, epidemiology and clinical aspects. London: Edward Arnold, 1985:20825.

3 Maxwell JD, Knapman P. Effect of coroners' rules on death certification for alcoholic liver disease. BrMed f 1985;291:708.

4 Barrison IG, Viola L, Murray-Lyon IM. Do housemen take an adequate drinking history? BrMed 7 1980;281:1040.

(Accepted 9 April 1987)

Academic Department of Psychiatry, University College and Middlesex School of Medicine, London W1N 8AA

GARY BELL, BA, MRCPSYCH, lecturer

Department of Psychiatry, Wexham Park Hospital, Slough SL2 4HL

ANNE CREMONA, MRCPSYCH, consultant

Correspondence to: Dr Bell.

\section{Doctors have no time for alcohol screening}

Despite the incidence of alcohol related problems in patients in general hospitals ${ }^{1}$ most of those who drink to excess are undetected because doctors fail to take accurate histories of alcohol intake. ${ }^{2}$ Evidence suggests that early identification of problem drinkers improves outcome and that brief interventions are beneficial and cost effective. ${ }^{3}$ We report our efforts to encourage junior medical staff to incorporate a few brief screening questions on alcohol intake into the medical histories they take from patients.

\section{Patients, methods, and results}

Doctors at York District Hospital were encouraged to administer brief alcohol screening questionnaires to patients admitted to nine medical and orthopaedic wards. The questionnaire incorporated items on regular consumption and binge drinking and included modified CAGE questions ${ }^{4}$ (copies available on request). Standard units of alcohol were used to measure consumption, one unit equalling half a pint of beer, lager, or cider; a single measure of spirits; a glass of wine; or a small glass of sherry. "Safe levels" were those recommended by the Health Education Council. ${ }^{5}$ Staff knew that some of the patients identified as at risk would be offered education about alcohol, the potential benefits of which would be evaluated subsequently.

In the first nine months of the study $32 \%$ of all patients admitted who were aged 16 and over were screened for alcohol problems $(n=1606)$. Of those screened, 90 (18\%) of the 514 men admitted to the medical wards and $74(20 \%)$ of the 367 men admitted to the orthopaedic wards were "positive" for alcohol related problems. The corresponding figures for women were $12 / 330(4 \%)$ and $8 / 395(2 \%)$, respectively. A sample of every fifth admission screened by a researcher (NR) confirmed these results.

There was no screening bias with regard to age or sex although there was with regard to specialty: $39 \%$ of patients admitted to the orthopaedic wards were screened compared with $28 \%$ of patients admitted to the medical wards. This difference is probably explained by the nature of the admissions, patients in orthopaedic wards generally having non-urgent conditions and therefore being easier to screen than patients on acute medical wards, where there may have been more pressure of time. This impression is confirmed by the poor screening rate in the only acute orthopaedic ward.

\section{Comment}

An accurate medical history is probably the doctor's best diagnostic tool. Brief screening questionnaires are more sensitive in detecting alcohol problems than laboratory tests and are quick and relatively cheap. ${ }^{3}$ The alcohol screening questionnaire suggested that $18-20 \%$ of the men admitted were problem drinkers. The doctors regarded the questionnaire as easy to administer, taking only one or two minutes to complete. Most believed that screening for alcohol problems was worth while and that the questionnaire facilitated this, although the questionnaire was administered to only a third of the patients admitted during the study period. The main reasons given for failure to screen were the patient's condition and pressure of time.

Some doctors screened most of the patients they admitted while others screened few or none at all. Although individual doctors were working on several wards, some of which were regarded as busier than others, their screening rates remained fairly constant, suggesting that the individual doctor's attitude to alcohol problems is an important factor in their 